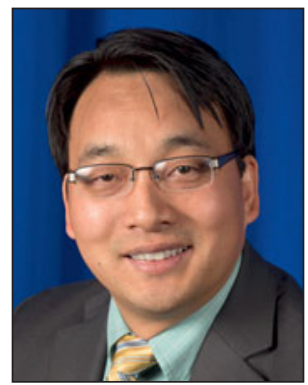

Jian Wang

Guest Editor for this issue of MRS Bulletin

Mechanical and Materials Engineering, University of Nebraska-Lincoln, USA; tel. 402-472-1514; and email jianwang@unl.edu.

Wang is an associate professor in the Department of Mechanical and Materials Engineering at the University of Nebraska-Lincoln. He received his $\mathrm{PhD}$ degree from Rensselaer Polytechnic Institute in 2006. He worked at Los Alamos National Laboratory (LANL) from 2006 to 2015. His research focuses on quantitative exploration of structure-property relationships of structural and nanostructured materials. He has published more than 170 journal articles. Wang's awards include the International Journal of Plasticity Award in 2015, the TMS Young Leader Professional Development Award in 2013, the LANL Early Career Award in 2011, and the LANL Distinguished Postdoctoral Performance Award in 2009.

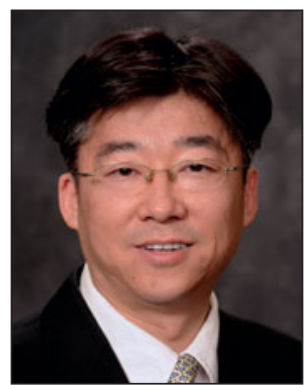

\section{Xinghang Zhang}

Guest Editor for this issue of MRS Bulletin

Departments of Mechanical Engineering and

Materials Science and Engineering, Texas

A\&M University, USA; tel. 979-845-2143;

and email zhangx@tamu.edu.

Zhang is a professor at Texas A\&M University. He received his BS degree from Jilin University, China, in 1995, MS degree from the Institute of Metals Research, China, and PhD degree from North Carolina State University in 2001, and was a director-funded postdoctoral fellow at Los Alamos National Laboratory. His research interests include synthesis and mechanical behavior of nanostructured metals, and radiation damage in nanomaterials. He has published more than 160 journal articles and holds two patents. Zhang's awards include a National Science Foundation Early Career Award.

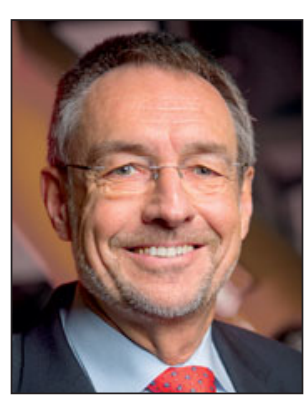

\section{Wolfgang Bleck}

Steel Institute, RWTH Aachen

University, Germany; email wolfgang.

bleck@iehk.rwth-aachen.de.

Bleck has been head of the Steel Institute at the RWTH Aachen University, Germany, since 1994, served as Vice Rector for Research from 2002 to 2005, and became dean of the faculty of Georesources and Materials Engineering in 2014. He studied physical metallurgy at the Clausthal University of Technology (TU Clausthal), earning a Dr.-Ing. degree in 1979. Bleck is active in the development and characterization of coldformable and high-strength steels, new processes for steel products, lightweight structures, principles of steel design, and numerical modeling of material and component properties.

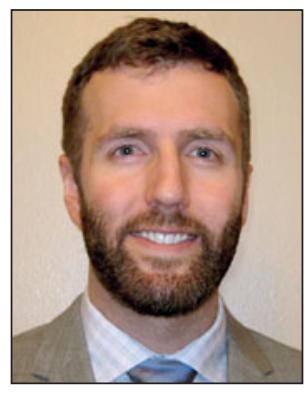

\section{Daniel C. Bufford}

Radiation-Solid Interactions Department,

Sandia National Laboratories, USA

email dcbuffo@sandia.gov.

Bufford has been a postdoctoral researcher at Sandia National Laboratories since receiving his $\mathrm{PhD}$ degree in materials science and engineering from Texas A\&M University in 2013. His research interests include the controlled synthesis of engineered grain-boundary structures in metals, and stability of these interfaces under different conditions. Of particular interest is the application of in situ transmission electron microscope techniques to reveal material behaviors at the nanoscale. Bufford received the 2013 Materials Research Society Graduate Student Silver Award.

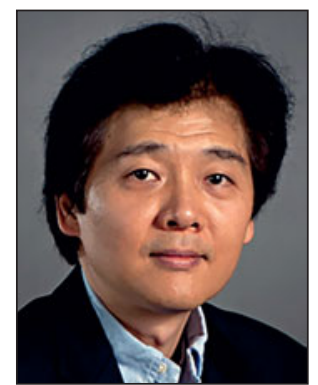

Ming Dao

Department of Materials Science and Engineering, Massachusetts Institute of Technology, USA; tel. 617-253-2100; and email mingdao@mit.edu.

Dao is a principal research scientist in the Department of Materials Science and Engineering, and serves as the principal investigator and director of the Nanomechanics Laboratory at the Massachusetts Institute of Technology. His research interests include nanostructured materials, contact mechanics, and biomaterials, as well as cell mechanics versus human diseases. Dao has published more than 110 journal papers.

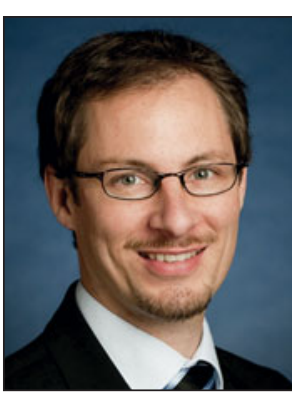

\section{Christoph Eberl}

Laboratory for Micro- and Materials Mechanics, Institute for Microsystems Technology, University of Freiburg, and Fraunhofer Institute for Mechanics of Materials, Germany; email chris.eber|@imtek. de and chris.eberl@iwm.fraunhofer.de. Eberl is a full professor at the Albert-LudwigsUniversität Freiburg (University of Freiburg) and a deputy director at the Fraunhofer Institute for Mechanics of Materials IWM. He received his engineering degree in 2001 and a $\mathrm{PhD}$ degree in 2004 in materials science from the University of Stuttgart, Germany. He worked as a postdoc at Johns Hopkins University and has maintained a visiting scientist position since 2007. Eberl's research focuses on the experimental characterization and modeling of deformation and degradation mechanisms in materials.

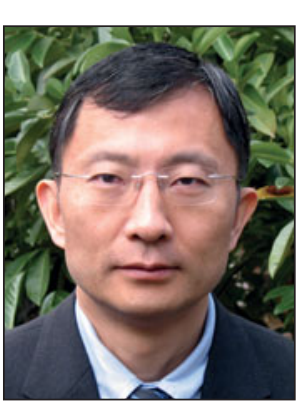

Huajian Gao

School of Engineering, Brown University,

USA; tel. 401-863-2626; and email huajian_gao@brown.edu.

Gao is the Walter H. Annenberg Professor of Engineering at Brown University. He earned his $\mathrm{PhD}$ degree in engineering science at Harvard University in 1988. He is a member of the National Academy of Engineering, and his awards include a John Simon Guggenheim Fellowship in 1995, the 2015 William Prager Medal from the Society of Engineering Science, and the 2015 Nadai Medal from the American Society of Mechanical Engineers.

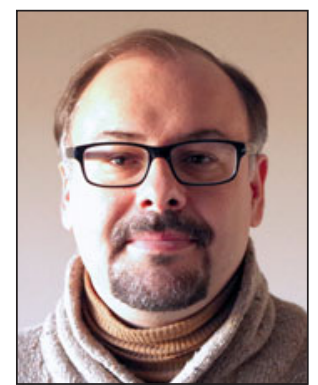

Ivan Gutierrez-Urrutia

National Institute for Materials Science, Japan; email gutierrezurrutia.ivan@nims.go.jp.

Gutierrez-Urrutia is a senior researcher at the National Institute for Materials Science (NIMS) He studied physics and earned a PhD degree in materials science and engineering from the University of the Basque Country, Spain, in 2003. In 2007, he joined the Max-Planck-Institut für Eisenforschung (Max Planck Institute for Iron Research) in Germany, where he worked as a scientist until 2014, when he joined NIMS Gutierrez-Urrutia's research interests include microstructure physics, deformation mechanisms, and electron microscopy. 


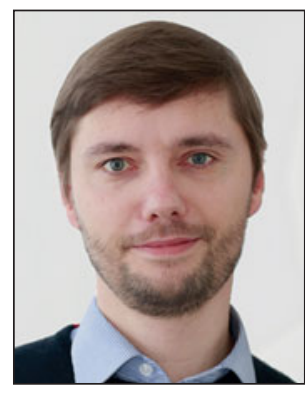

Tilmann Hickel

Department of Computational Materials

Design, Max-Planck-Institut für Eisenforschung

(Max Planck Institute for Iron Research),

Germany; tel. +49-211 6792-575;

and email t.hickel@mpie.de.

Hickel became head of the Computational Phase Studies Group within the Computational Materials Design Department at the Max-PlanckInstitut für Eisenforschung (institute for iron and steel research) in 2006. He studied physics at Humboldt University of Berlin, Germany, and Loughborough University, UK, and earned his $\mathrm{PhD}$ degree in 2005 on many-body theories for magnetic materials. Hickel's group focuses on ab initio-based simulation of materials properties and phase stabilities at finite temperatures.

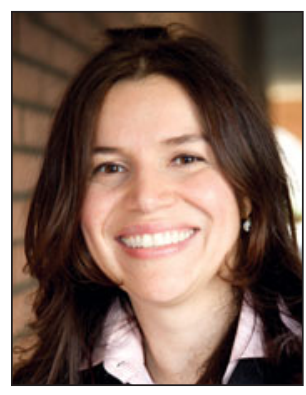

\section{Andrea Maria Hodge}

Mork Family Department of Chemical

Engineering and Materials Science, University of Southern California, USA; tel. 213-740-4225; and email ahodge@usc.edu.

Hodge is a professor in the Mork Family Department of Chemical Engineering and Materials at the University of Southern California (USC). She earned her PhD degree in materials science and engineering from Northwestern University in 2002. Prior to joining USC in August 2007, she was a staff scientist at Lawrence Livermore National Laboratory. Hodge's research focuses on the processing and mechanical behavior of engineered materials for advanced applications at the nano- and microscale.

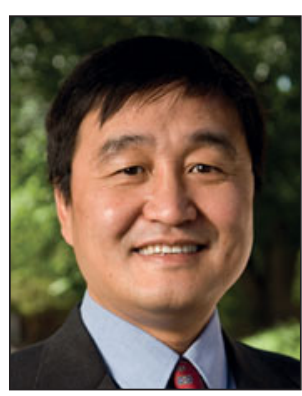

Yanyao Jiang

Mechanical Engineering Department,

University of Nevada, Reno, USA; tel. 775-

784-4510; and email yjiang@unr.edu.

Jiang is a professor in the Mechanical Engineering Department at the University of Nevada, Reno. He received his BS degree in mechanical engineering from Northeastern University, China, his MS degree in solid mechanics from Zhejiang University, China, and his PhD degree in mechanical engineering from the University of Illinois at Urbana-Champaign. His research interests focus on the experimental exploration and theoretical modeling of mechanical behavior of materials and structures, including cyclic plasticity, fatigue, and fracture.

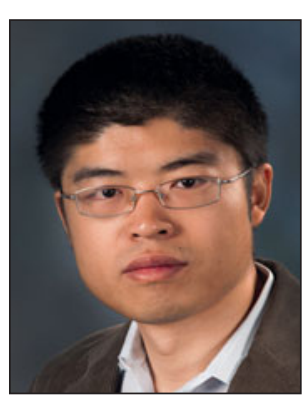

\section{Nan Li}

Center for Integrated Nanotechnologies,

Los Alamos National Laboratory, USA;

tel. 505-665-1857; and email nanli@lanl.gov.

$\mathrm{Li}$ is a materials scientist at Los Alamos National Laboratory (LANL). He received his $\mathrm{PhD}$ degree from Texas A\&M University in 2010. His research focuses on designing materials with enhanced mechanical performance and radiation tolerance through the control of microstructure and defect phenomena. He was awarded the The Minerals, Metals and Materials Society Young Leader Professional Development Award in 2014, LANL Distinguished Postdoctoral Performance Award in 2011, ACTA Student Award in 2010, and TMS Best Graduate Student Paper Award in 2009.

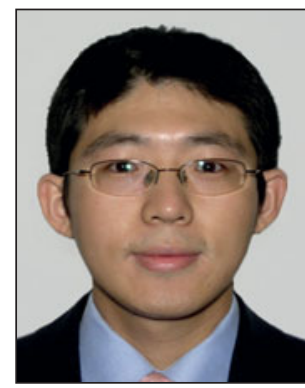

Xiaoyan Li

Centre for Advanced Mechanics and Materials, Applied Mechanics Laboratory, Department of Engineering Mechanics, Tsinghua University,

China; email xiaoyanlithu@tsinghua.edu.cn.

$\mathrm{Li}$ is an associate professor in the Department of Engineering Mechanics at Tsinghua University. He received his $\mathrm{PhD}$ degree from Brown University in 2012. His research interests focus on deformation and fracture of nanostructured materials, such as nanocrystalline/nanotwinned materials and low-dimensional nanomaterials.

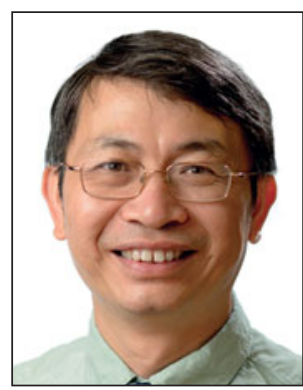

\section{Xiaozhou Liao}

School of Aerospace, Mechanical and

Mechatronic Engineering, The University of Sydney, Australia; tel. 6129351 2348;

and email xiaozhou.liao@sydney.edu.au.

Liao is a professor in the School of Aerospace, Mechanical and Mechatronic Engineering at The University of Sydney. He received his PhD degree for electron microscopy investigations of semiconductor quantum dots from The University of Sydney in 2000. He then moved to Los Alamos National Laboratory as a directorfunded postdoctoral fellow in 2001, and was a research scientist at The University of Chicago from 2004 to 2006. Liao returned to Sydney as a lecturer in 2006. His research focuses on electron microscopy characterization of advanced materials.

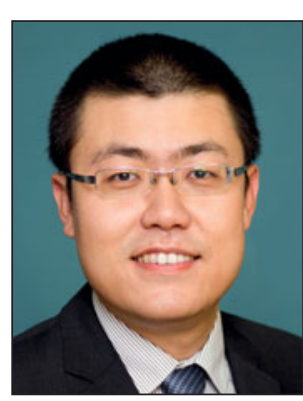

Yue Liu

Materials Science and Technology Division,

Los Alamos National Laboratory, USA;

tel. 505-665-9336; and email yueliu@lanl.gov.

Liu is a postdoctoral research associate at Los Alamos National Laboratory. He received his BS degree in microelectronics engineering from Fudan University, China, MS degree in mechanical engineering, and $\mathrm{PhD}$ degree in materials science and engineering from Texas A\&M University. His research interests focus on the synthesis and microstructure characterization of materials with desired physical properties, often under extreme temperature, pressure, radiation, and corrosion environments. Liu has co-authored over 30 journal publications.

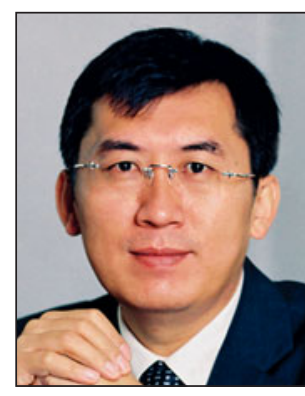

Ke Lu

Institute of Metal Research, Chinese Academy of Sciences, China; tel. +86-24-2390-6826; and email lu@imr.ac.cn.

$\mathrm{Lu}$ is a professor and director of Shenyang National Laboratory for Materials Science, Institute of Metal Research, Chinese Academy of Sciences. His research interests focus on nanostructured metals and alloys, including those with nanograined, nanotwinned, nanolaminated, and gradient nanograined structures. His research focuses on the synthesis and processing, structure characterization, phase transformation, and mechanical properties of nanostructured metals. Lu is a Fellow of MRS. 


\section{MEET OUR AUTHORS}

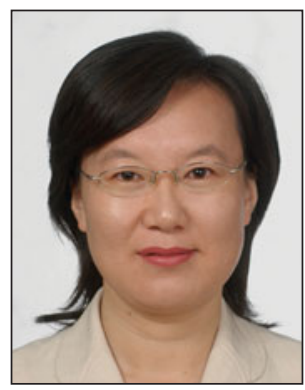

Lei Lu

Institute of Metal Research, Chinese Academy of Sciences, China; email llu@imr.ac.cn.

Lu is a professor in the Institute of Metal Research, Chinese Academy of Sciences. She is a member of the International Community of Nanostructured Materials. Her research focuses on the synthesis, microstructure characterization, and mechanical properties of nanostructured metallic materials. She has authored or co-authored more than 80 journal publications. Lu holds six domestic patents and three international patents, and has presented more than 30 invited lectures at international conferences.

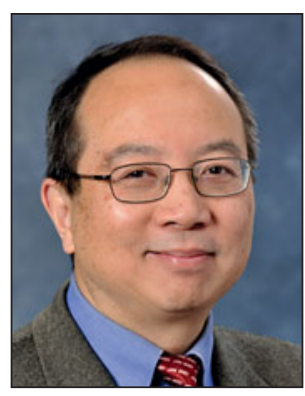

\section{Scott X. Mao}

Department of Mechanical Engineering

and Materials Science, University of

Pittsburgh, USA; tel. 412-624-9602

and emailsxm2@pitt.edu.

Mao is the William Kepler Whiteford Chair Professor in the Department of Mechanical Engineering and Materials Science at the University of Pittsburgh. He received his BSc degree from Beijing University of Aeronautics and Astronautics, PhD degree from Tohoku University with the completion of his dissertation at the Massachusetts Institute of Technology (MIT) in 1988, and worked in MIT and Harvard University as a postdoctoral researcher and visiting faculty. In 2006, he was awarded the Chancellor's Distinguished Research Award. Mao has 250 publications on topics such as in situ high-resolution transmission electron microscopy, mechanical behavior of materials, fracture mechanics, and nanocrystalline and single-element metallic glasses.

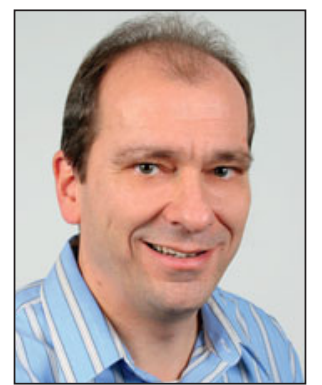

\section{Joachim Mayer}

Central Facility for Electron Microscopy,

RWTH Aachen University, and Ernst

Ruska-Centre, Forschungszentrum Jülich,

Germany; email mayer@gfe.rwth-aachen.de.

Mayer has been professor and head of the Central Facility for Electron Microscopy at RWTH Aachen University since 1999. In 2004, he received a co-appointment as one of two directors of the newly founded Ernst Ruska-Centre, a German national user facility jointly founded by the Research Centre Jülich and RWTH Aachen University. He studied physics at the University of Stuttgart and received his PhD degree in physics at the Max-Planck-Institut für Metallforschung (Max Planck Institute for Intelligent Systems), Germany, in 1988. His research focuses on the application of new methods in electron microscopy in the areas of materials science, nanoelectronics, and energy systems.

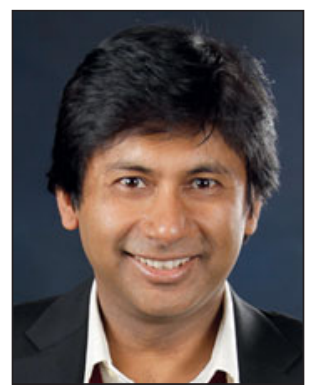

\section{Amit Misra}

Department of Materials Science and Engineering, University of Michigan, USA; tel. 734-763-2445; and emailamitmis@umich.edu.

Misra has been a professor and chair of the Department of Materials Science and Engineering at the University of Michigan since June 2014. From 1996 to 2014, he worked at Los Alamos National Laboratory, New Mexico. He received his educational training from the Indian Institute of Technology, Banaras Hindu University, India, for his undergraduate degree and the University of Michigan for his graduate degree. His research interests include materials processing, nanomechanics, defects and interfaces in materials, and transmission electron microscopy. Misra has co-authored more than 275 peer-reviewed publications and is a member of the MRS Bulletin editorial board.

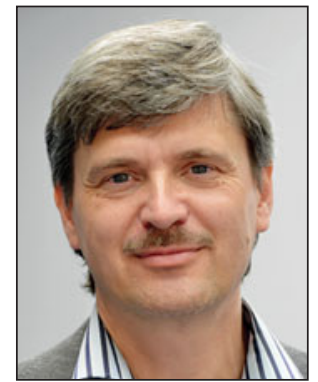

Jörg Neugebauer

Max-Planck-Institut für Eisenforschung

Germany; email neugebauer@mpie.de.

Neugebauer is director of the Max-Planck-Institut

für Eisenforschung (institute for iron and steel research) and a professor at the Universities of Paderborn and Bochum. He obtained his PhD degree in 1989 at the Humboldt University of Berlin, Germany. In 1996, he became a senior research assistant at the Fritz-Haber-Institut Berlin, and in 1998, head of an independent Max Planck research group at the same institute. Neugebauer's interests include the development and application of ab initio scale-bridging computer simulations to describe complex functional and structural materials in realistic environments.

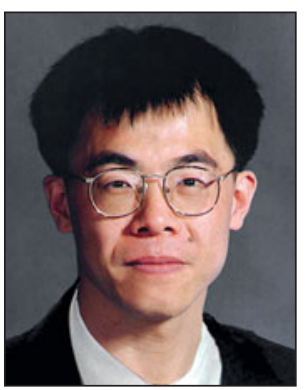

\section{Jianfeng Nie}

Department of Materials Science

and Engineering, Monash University,

Australia; tel. 6139905 9605; and

email jianfeng.nie@monash.edu.

Nie is a professor in the Department of Materials Science and Engineering at Monash University. He received his PhD degree from Monash University in 1993. He joined both The University of Queensland and the Commonwealth Scientific and Industrial Research Organisation as a postdoctoral fellow, and returned to Monash in 1995. Nie's research interests include physica metallurgy of light alloys, crystallography of solid-solid phase transformations, applications of transmission electron microscopy, and processing-microstructureproperty relationships.

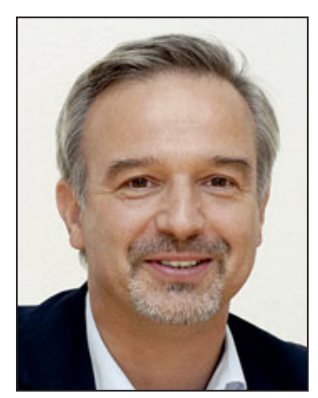

Dierk Raabe

Department of Microstructure Physics and Alloy Design, Max-Planck-Institut für Eisenforschung, RWTCH Aachen University, Germany; tel. +49(0)211-6792-340; and email d.raabe@mpie.de. Raabe has been director of the Max-PlanckInstitut für Eisenforschung (institute for iron and steel research) since 1999, and is also professor at Rheinisch-Westfälische Technische Hochschule (RWTH) Aachen University. He earned his PhD degree in 1992, and then received his Habilitation degree at RWTH Aachen in physical metallurgy and metal physics in 1997. Between 1997 and 1999, he worked in the Materials Science and Engineering Department at Carnegie Mellon University and at the National High Magnetic Field Laboratory in Tallahassee, Fla. Raabe's interests include alloy design, computational materials science, crystal plasticity, and atom probe tomography.

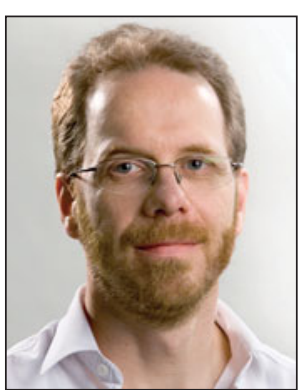

\section{Franz Roters}

Department of Microstructure Physics and Alloy Design, Max-Planck-Institut für Eisenforschung, Germany; email f.roters@mpie.de.

Roters has been group leader at the Max-PlanckInstitut für Eisenforschung (institute for iron and steel research) since 2000. He studied physics at TU Braunschweig. He earned his PhD degree in 1999 and his Habilitation degree in computational materials science in 2011 , both at RWTH Aachen. He then became a postdoctora lecturer in Aachen. From 1999 to 2000, Roters worked at the Research and Development Department of VAW Aluminium in Bonn. His interests include computational materials science and crystal plasticity. 


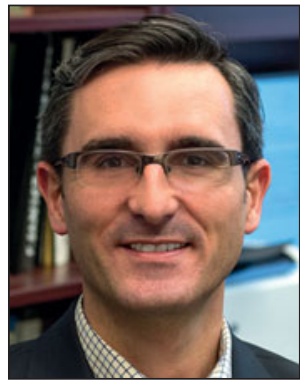

Frederic Sansoz

School of Engineering, The University of Vermont, USA; tel. 802-656-3837; and email frederic.sansoz@uvm.edu. Sansoz is a professor of mechanical engineering and materials science at The University of Vermont. He earned his BS degree in mechanical and aerospace engineering and MS degree in materials science and engineering from the École Nationale Supérieure de Mécanique et Aérotechnique in France in 1996, and a PhD degree in materials science and engineering from the École Nationale Supérieure des Mines de Paris in 2000. His research focuses on understanding the mechanics and physics of interface-dominated nanomaterials using materials theory, atomistic computer simulations, and nanoscale experiments. Sansoz received the NSF CAREER Award in 2008.

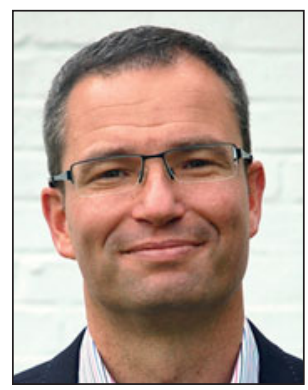

Jochen M. Schneide

RWTH Aachen University, Germany;

email schneider@mch.rwth-aachen.de.

Schneider is a professor of materials chemistry at RWTH Aachen University. He received his $\mathrm{PhD}$ degree in surface engineering from Hull University, UK. His research interests include the materials science of thin films grown by plasma-assisted vapor deposition. He was awarded the Sofya Kovalevskaya Prize by the Alexander von Humboldt Foundation for excellence in thin-film materials science research, and was named a Fellow of AVS in 2013 and Max Planck in 2015.

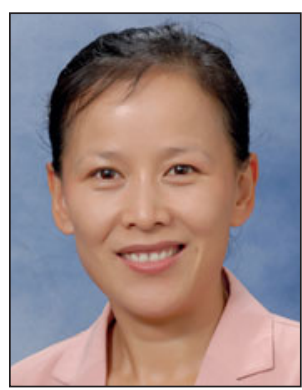

\section{Haiyan Wang}

Department of Electrical and Computer Engineering, Texas A\&M University,

USA; tel. 979-845-5082; and

email wangh@ece.tamu.edu.

Wang is a full professor in the Department of Electrical and Computer Engineering at Texas A\&M University. She received her PhD degree from North Carolina State University in 2002. She was promoted to associate professor and full professor at Texas A\&M in 2010 and 2014, respectively. Wang has received several awards, including becoming an ACerS Fellow in 2015, an ASM Fellow in 2014, and the TAMEST O'Donnell Award in 2015.

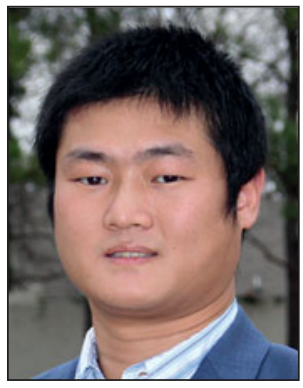

\section{Jiangwei Wang}

Center of Electron Microscopy,

School of Materials Science and

Engineering, Zhejiang University, China;

email jiangwei_wang@zju.edu.cn.

Wang joined the faculty of the Center of Electron Microscopy, School of Materials Science and Engineering at Zhejiang University in 2016. He received his $\mathrm{PhD}$ degree from the University of Pittsburgh in 2014. His research interests include nanomechanics, in situ characterization of energy-storage materials, and in situ TEM technique. Wang received the MRS Graduate Student Silver Award at the 2014 MRS Spring Meeting and co-organized a seminar at the 2016 TMS Annual Meeting \& Exhibition.

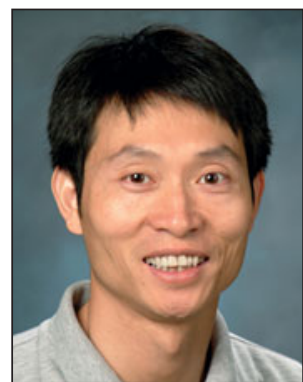

\section{Y. Morris Wang}

Lawrence Livermore National Laboratory,

USA; email wang35@|Inl.gov.

Wang is a staff scientist at Lawrence Livermore National Laboratory and a founding member of the Nanoscale Synthesis and Characterization Lab. He received two master's degrees and his $\mathrm{PhD}$ degree in 2003 from Johns Hopkins University. His current research interests include mechanical behavior of nanostructured metals, additive manufacturing, lithium-ion batteries, energy conversion, and fusion targets. Wang is an APS Fellow, and is a recipient of the Harold Graboske Jr. Fellowship and the Nano 50 Innovator Award.

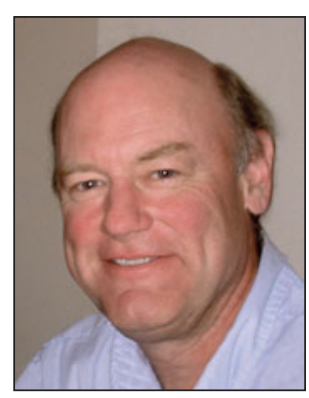

\section{James Wittig}

Vanderbilt University, USA; email

j.wittig@vanderbilt.edu.

Wittig has been on the faculty at Vanderbilt University since 1987, and is currently the director of the Electron Optics Laboratory in the Vanderbilt Institute for Nanoscale Science and Engineering. He received his $\mathrm{PhD}$ degree in materials science and engineering from Stanford University in 1985. Between 1985 and 1987, he worked at Max-Planck-Institut für Eisenforschung (Max Planck Institute for Iron Research), Germany. Wittig's research includes studying the deformation mechanisms in metal alloys using transmission electron microscopy

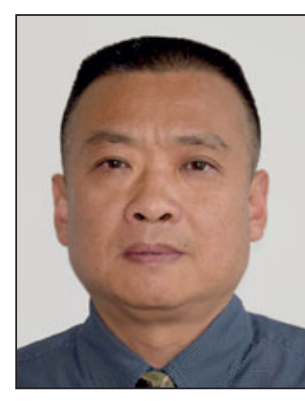

\section{Peidong Wu}

Department of Mechanical Engineering, McMaster University, Canada;

tel. 1-905-525-9140 (x20092); and

email peidong@mcmaster.ca.

Wu is a professor in the Department of Mechanical Engineering at McMaster University. He received his BSc degree from Zhejiang University, MSc degree from China University of Mining and Technology, and $\mathrm{PhD}$ degree from Delft University of Technology. His research interests focus on constitutive modeling of large-strain behavior of engineering materials.

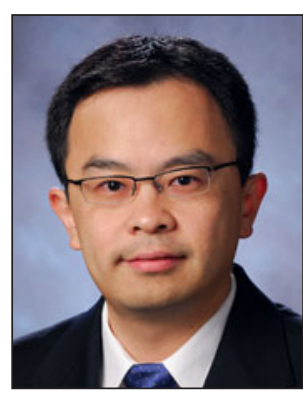

\section{Ting Zhu}

George W. Woodruff School of Mechanical

Engineering, Georgia Institute of

Technology, USA; tel. 404-894-6597;

and email ting.zhu@me.gatech.edu.

Zhu is a professor in the George W. Woodruff School of Mechanical Engineering at the Georgia Institute of Technology (Georgia Tech). He received his $\mathrm{PhD}$ degree in mechanical engineering from the Massachusetts Institute of Technology in 2004. He worked as a postdoc at Harvard University before joining Georgia Tech in 2005. His research is focused on mechanics and materials modeling. He received the ASME Sia Nemat-Nasser Early Career Award in 2013 and the Young Investigator Medal from the Society of Engineering Science in 2014. Zhu is an ASME Fellow.

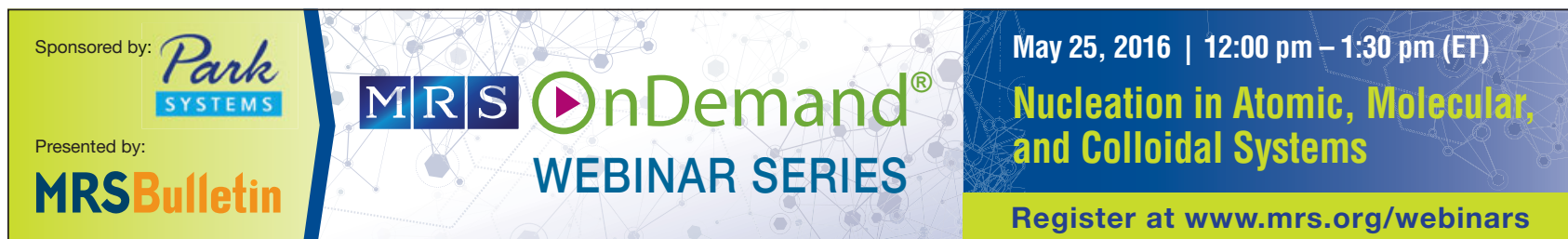

